\title{
DYNAMICS OF THE DEEP SOLAR PHOTOSPHERE AT SUBGRANULAR SCALES
}

\author{
A. NESIS, R. HAMMER, M. KIEFER, AND H. SCHLEICHER \\ Kiepenheuer-Institut für Sonnenphysik \\ Schöneckstr. 6, D-79104 Freiburg, F.R.G.
}

\section{Introduction}

Extending our previous studies of the dynamics of solar granulation (Nesis et al., 1997) we investigated the relationship between granular flow and the emergence of turbulence in the deep photosphere. Our main goal is to explore if such a relationship exists, and if so, to define it quantitatively. To this end we take advantage of the excellent signal approximation property of wavelets. The material for the present work is a series of spectrograms of high spatial resolution covering a time span of $12 \mathrm{~min}$. They were taken at the center of the solar disk with the German Vacuum Tower Telescope in Izaña (Tenerife, Spain) in 1994, and include several absorption lines of different strengths; for more details see Nesis et al. (1997). The spectrograms were digitized and processed with wavelet techniques and regression analysis, in order to investigate the granular convective flow, the associated turbulence, and their mutual connection.

\section{Results and Conclusions}

Figure 1. shows the traces of the Doppler velocity (full line) and the turbulent velocity (dotted line) along the spectrograph slit. The Doppler velocity corresponds to the Doppler shift of the line core, whereas the turbulent velocity is reflected by the fwhm of the line. We found that granular flow speed and turbulence cannot be related by a regression line; rather the convective flow and the turbulence appear to be related by an attractor in the convective flow speed-turbulence phase space. The behavior of the regression analysis follows closely the history of the granulation dynamics over the entire $12 \mathrm{~min}$ time span, which corresponds roughly to a mean turn-over time of a granule (Mehltretter et al., 1978). Thus, we tend to assert that convective flow and turbulence can be interpreted in terms of a dynamical system, which is shown 


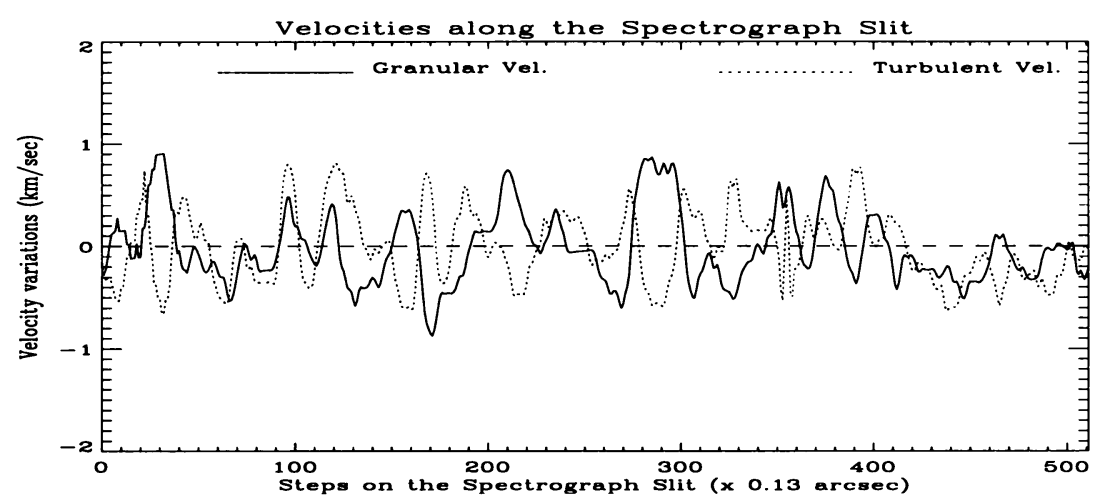

Figure 1. Doppler velocity and turbulent velocity.

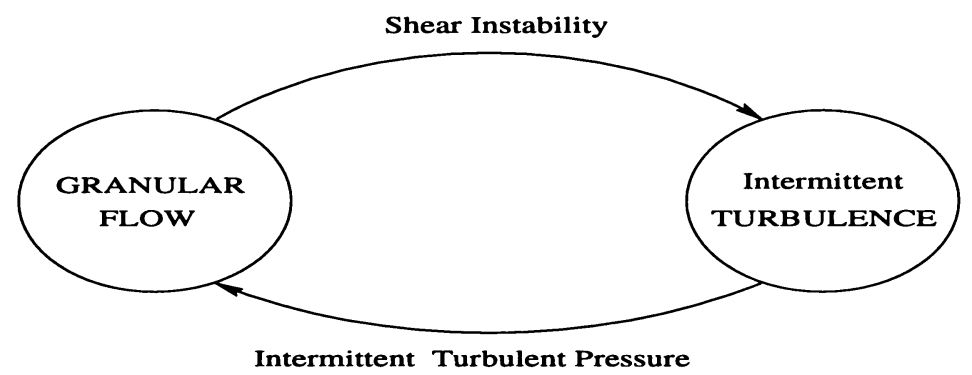

At locations of steep gradients in the granular flow patterns, shear instability produces turbulence. The associated turbulent pressure will backreact on the convective flow.

Figure 2. The dynamical system.

in Fig. 2. The calculated turbulent pressure shown in Table 1 supports this assertion.

TABLE 1. Hydrodynamical implications of our observations.

\begin{tabular}{lllll}
\hline $\mathrm{Re}$ & $\nu_{t u r b}$ & $\mathrm{P}_{\text {turb }}$ & $\mathrm{P}_{\text {turb }} / \mathrm{P}_{\text {gas }}$ & all values at \\
\hline $10^{9}$ & $10^{8} \mathrm{~m}^{2} \mathrm{~s}^{-1}$ & $\leq 10^{4} \mathrm{~Pa}$ & $\leq 0.5$ & $\approx \tau_{5000}=1$ \\
\hline
\end{tabular}

\section{References}

Nesis, A., Hammer, R., Hanslmeier, H., Schleicher, H., Sigwarth, M., and Staiger, J. (1997), Astronomy and Astrophysics Vol. 326, p. 851

Mehltretter, P. (1978), Astronomy and Astrophysics Vol. 62, p. 3 